\title{
MULTILEVEL LINEAR SAMPLING METHOD FOR INVERSE SCATTERING PROBLEMS*
}

\author{
JINGZHI LI ${ }^{\dagger}$, HONGYU LIU ${ }^{\ddagger}$, AND JUN ZOU ${ }^{\dagger}$
}

\begin{abstract}
A novel multilevel algorithm is presented for implementing the widely used linear sampling method in inverse obstacle scattering problems. The new method is shown to possess asymptotically optimal computational complexity. For an $n \times n$ sampling mesh in $\mathbb{R}^{2}$ or an $n \times n \times n$ sampling mesh in $\mathbb{R}^{3}$, the proposed algorithm requires one to solve only $\mathcal{O}\left(n^{N-1}\right)$ far-field equations for a $\mathbb{R}^{N}$ problem $(\mathrm{N}=2,3)$, and this is in sharp contrast to the original version of the method which needs to solve $n^{N}$ far-field equations. Numerical experiments are presented to illustrate the promising feature of the algorithm in significantly reducing the computational cost of the linear sampling method.
\end{abstract}

Key words. multilevel linear sampling method, inverse scattering problems, optimal computational complexity

AMS subject classifications. 78A45, 35R30

DOI. $10.1137 / 060674247$

1. Introduction. In their original work [5], Colton and Kirsch developed a "simple" method for the shape reconstruction in inverse scattering problems which is nowadays known as the linear sampling method (LSM). The method has been extensively studied and extended in several directions; we refer the reader to [8] and [12] for a comprehensive review. The current work is mainly concerned with an implementation technique of the LSM. We take as our model problem the inverse acoustic sound-soft obstacle scattering by time-harmonic plane waves. But like the original LSM, our algorithm can be equally applied to other inverse problems, such as the acoustic sound-hard obstacle scattering or electromagnetic obstacle scattering.

Consider a sound-soft scatterer $\mathbf{D}$, which is assumed to be the open complement of an unbounded domain of class $C^{2}$ in $\mathbb{R}^{N}(N=2,3)$, that is, we include scattering from more than one (but finitely many) component obstacle in our analysis. Given an incident field $u^{i}$, the presence of the obstacle will give rise to a scattered field $u^{s}$. Throughout, we take $u^{i}(x)=\exp \{\mathrm{i} k x \cdot d\}$ to be a time-harmonic plane wave, where $\mathrm{i}=\sqrt{-1}, d \in \mathbb{R}^{N-1}$, and $k>0$ are, respectively, the incident direction and wave number. We define $u(x)=u^{i}(x)+u^{s}(x)$ to be the total field, which satisfies the following Helmholtz system (cf. [6], [7]):

$$
\left\{\begin{array}{lr}
\Delta u+k^{2} u=0 & \text { in } \mathbb{R}^{N} \backslash \overline{\mathbf{D}}, \\
u(x)=0 & \text { on } \partial \mathbf{D}, \\
\lim _{r \rightarrow \infty} r^{(N-1) / 2}\left(\frac{\partial u^{s}}{\partial r}-\mathrm{i} k u^{s}\right)=0,
\end{array}\right.
$$

where $r=|x|$ for any $x \in \mathbb{R}^{N}$. The direct problem (1.1) has been well understood, and it is known that there exists a unique solution $u \in C^{2}\left(\mathbb{R}^{N} \backslash \overline{\mathbf{D}}\right) \cap C\left(\mathbb{R}^{N} \backslash \mathbf{D}\right)$. Moreover,

${ }^{*}$ Received by the editors November 7, 2006; accepted for publication (in revised form) November 15, 2007; published electronically March 21, 2008.

http://www.siam.org/journals/sisc/30-3/67424.html

${ }^{\dagger}$ Department of Mathematics, The Chinese University of Hong Kong, Shatin, N.T., Hong Kong (jzli@math.cuhk.edu.hk, zou@math.cuhk.edu.hk). The third author's work was substantially supported by Hong Kong RGC grants 404105 and 404606.

$\ddagger$ Department of Mathematics, University of Washington, Box 354350, Seattle, WA 98195 (hyliu@ math.washington.edu). 
the asymptotic behavior at infinity of the scattered wave $u^{s}$ is governed by

$$
u^{s}(x)=\frac{e^{i k|x|}}{|x|^{(N-1) / 2}}\left\{u_{\infty}(\hat{x})+\mathcal{O}\left(\frac{1}{|x|}\right)\right\} \quad \text { as }|x| \rightarrow \infty,
$$

uniformly for all directions $\hat{x}=x /|x| \in \mathbb{S}^{N-1}$. The analytic function $u_{\infty}(\hat{x})$ is defined on the unit sphere $\mathbb{S}^{N-1}$ and called the far-field pattern (see [7]). We shall write $u_{\infty}(\hat{x} ; \mathbf{D}, d, k)$ to specify its dependence on the observation direction $\hat{x}$, the obstacle $\mathbf{D}$, the incident direction $d$, and the wave number $k$. The inverse obstacle scattering problem is to determine $\partial \mathbf{D}$ from the measurement of $u_{\infty}(\hat{x} ; d, k)$ for $\hat{x}, d \in \mathbb{S}^{N-1}$ and fixed $k>0$. This problem has been playing an indispensable role in many areas of sciences and technology such as radar and sonar, medical imaging, geophysical exploration, and nondestructive testing (see, e.g., [7]). Next, we shall give a brief description of the LSM for solving this important inverse problem.

First, we introduce the far-field operator $F: L^{2}\left(\mathbb{S}^{N-1}\right) \mapsto L^{2}\left(\mathbb{S}^{N-1}\right)$ defined by

$$
(F g)(\hat{x}):=\int_{\mathbb{S}^{N-1}} u_{\infty}(\hat{x}, d) g(d) d s(d), \quad \hat{x} \in \mathbb{S}^{N-1} .
$$

The LSM uses $g$ as an indicator and solves the following far-field equation:

$$
(F g)(\hat{x})=\Phi_{\infty}(\hat{x}, z), \quad \hat{x} \in \mathbb{S}^{N-1}, z \in \mathbb{R}^{N},
$$

where

$$
\Phi_{\infty}(\hat{x}, z)=\gamma \exp \{-\mathrm{i} k \hat{x} \cdot z\}
$$

with $\gamma=1 / 4 \pi$ in $\mathbb{R}^{3}$ and $\gamma=e^{\mathrm{i} \pi / 4} / \sqrt{8 \pi k}$ in $\mathbb{R}^{2}$. The following theorem forms the basis of the LSM (see, e.g., Theorem 4.1 in [8]).

Theorem 1.1. Assume that $k^{2}$ is not a Dirichlet eigenvalue for $-\Delta$ in $\mathbf{D}$. Then the following hold:

1. For $z \in \mathbf{D}$ and a fixed $\varepsilon>0$ there exists a $g_{\varepsilon}^{z} \in L^{2}\left(\mathbb{S}^{N-1}\right)$ such that

$$
\left\|F g_{\varepsilon}^{z}-\Phi_{\infty}(\cdot, z)\right\|_{L^{2}\left(\mathbb{S}^{N-1}\right)}<\varepsilon
$$

and

$$
\lim _{z \rightarrow \partial \mathbf{D}}\left\|g_{\varepsilon}^{z}\right\|_{L^{2}\left(\mathbb{S}^{N-1}\right)}=\infty
$$

2. For $z \in \mathbb{R}^{N} \backslash \overline{\mathbf{D}}$ and any given $\varepsilon>0$, every $g_{\varepsilon}^{z} \in L^{2}\left(\mathbb{S}^{N-1}\right)$ that satisfies

$$
\left\|F g_{\varepsilon}^{z}-\Phi_{\infty}(\cdot, z)\right\|_{L^{2}\left(\mathbb{S}^{N-1}\right)}<\varepsilon
$$

ensures

$$
\lim _{\varepsilon \rightarrow 0}\left\|g_{\varepsilon}^{z}\right\|_{L^{2}\left(\mathbb{S}^{N-1}\right)}=\infty .
$$

The LSM elegantly turns the reconstruction of the shape of obstacle $\mathbf{D}$ into the process of numerically determining the indicator function $g^{z}$ in Theorem 1.1. The general procedure is stated as follows (see also Chapter 4 in [3]).

\section{Algorithm LSM.}

1. Select a mesh $\mathcal{T}_{h}$ of sampling points in a region $\Omega$ which contains $\mathbf{D}$.

Copyright (C) by SIAM. Unauthorized reproduction of this article is prohibited. 
2. Use the Tikhonov regularization and the Morozov discrepancy principle to compute an approximate solution $g^{z}$ to the far-field equation (1.4) for each mesh point $z$ of $\mathcal{T}_{h}$.

3. Select a cut-off value $c$; then, count $z \in \mathbf{D}$ if $\left\|g^{z}\right\|_{L^{2}\left(\mathbb{S}^{N-1}\right)} \leq c$ and $z \notin \mathbf{D}$ if $\left\|g^{z}\right\|_{L^{2}\left(\mathbb{S}^{N-1}\right)}>c$.

A mathematical justification was given in [1] for the use of the LSM to determine $\mathbf{D}$ through the information of the indicator function $g^{z}$. The LSM has been proven to be numerically very successful and shown to possess several remarkable merits; see [8], [12], and the references therein. In fact, it has become one of the most important reconstruction algorithms in inverse obstacle scatterings. But there are also some drawbacks for the method; e.g., it fails to work when meeting some interior eigenvalue problem, and there is no standard strategy to choose the cut-off values; see [3]. Though the computational complexity is also one of its drawbacks, it is considerably more efficient and less computationally expensive than many other nonlinear methods such as the one based on the Lippmann-Schwinger equation (cf. [10]). Nevertheless, the LSM can still be very expensive in numerical implementations, as one has to solve the far-field equation (1.4) for each sampling point. There are several works which attempt to either circumvent the cost of sampling or improve the image results; see, e.g., [2], [4]. In the present work, we shall make an effort to reduce the computational complexity of the method. As one can see, for an $\mathbb{R}^{2}$ problem, by using an $n \times n$ mesh, we have to solve one linear integral equation (1.4) at each mesh point, which amounts to $n^{2}$ totally, while it is $n^{3}$ in the $\mathbb{R}^{3}$ case. And the computational counts can be huge in certain particular situations such as the following: When little a priori information on $\mathbf{D}$ is available, the initial guess region $\Omega$ should be chosen to be moderately larger than $\mathbf{D}$. In order to achieve a high-resolution reconstruction of $\mathbf{D}$, one needs a very fine mesh over $\Omega$, thus leading to a very large $n$. When the scatterer consists of multiple component obstacles with the distance between each two being several times larger than the sizes or diameters of the two obstacles, the initial region $\Omega$ is then required to contain all these components, which means that $\Omega$ must be chosen to be much larger than actually needed. Our main goal in this paper is to provide a fast numerical procedure to implement the LSM, and thus further consolidate its effectiveness, and, more importantly, to rigorously justify that the numerical algorithm has optimal computational complexity in terms of the mesh size $n$. To the best of our knowledge, this important issue on computational complexity of the LSM has not been seriously investigated yet.

In the next section, we will address the motivations and implementation details of the new algorithm and then prove its asymptotically optimal computational complexity. In section 3, some numerical experiments are performed to illustrate the promising feature of the algorithm in significantly reducing the computational cost of the LSM.

2. Multilevel linear sampling method. In this section, we will present a multilevel linear sampling method (MLSM), together with some theoretical analysis. For the sake of simplicity, we will carry out our discussion in $\mathbb{R}^{2}$, but all of the subsequent results can be straightforwardly extended to the three-dimensional case.

Let $\mathbf{D} \subset \mathbb{R}^{2}$ be a bounded domain as shown in Figure 1 (top-left) and suppose that we are going to use an $n \times n$ mesh for the LSM with $(n-1)^{2}$ cells of equal size. Clearly, in order to get some satisfactory reconstruction for the profile of $\mathbf{D}$, the mesh must be moderately fine in some sense. However, by performing the LSM on this fine mesh, we have to spend considerable computational cost in finding the indicator 

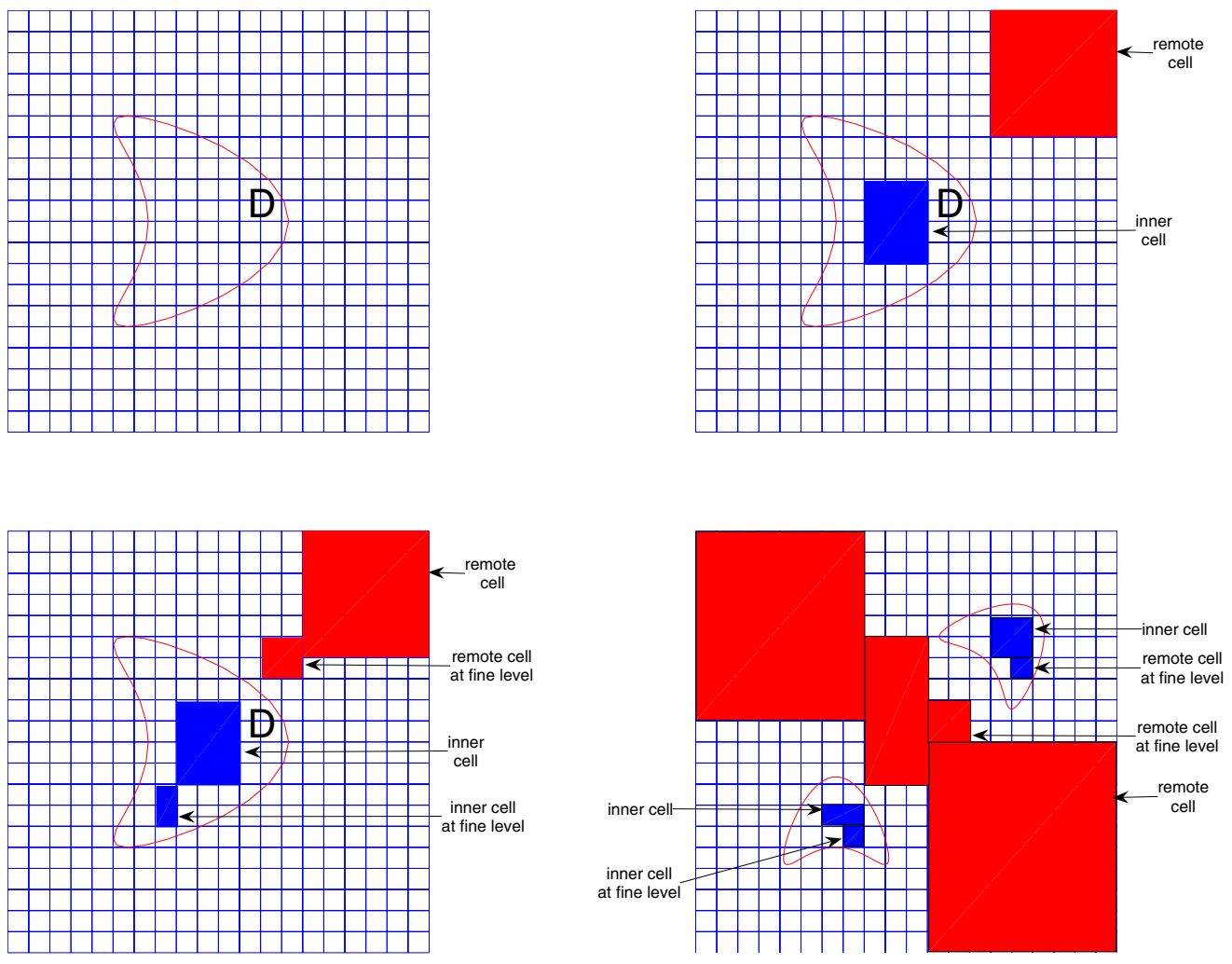

FIG. 1. Label-and-remove scheme.

functions in those "remote" cells which are far away from the scatterer $\mathbf{D}$, or in those "inner" cells which lie deeply inside $\mathbf{D}$; e.g., see the red and blue colored regions in Figure 1 (top-right). So, it would be very advantageous if we could get rid of the remote and inner cells in our computations. This can be naturally realized with a coarser mesh. In fact, this is reasonable since the indicator function $g^{z}$ has very large norms for $z$ in those remote cells while very small norms for $z$ in those inner cells. Moreover, it is noted that the cut-off value $c$ for the LSM in the fine mesh is still applicable on the coarsened mesh. Here, we would like to remark that, as pointed out in [3], the choice of $c$ is rather heuristic and there is still no standard strategy for it. To be more precise, we first choose a coarse grid covering the sampling region $\Omega$ and perform the LSM on this coarse level. Then based on the results of the LSM we will label and remove those remote and inner cells. Then, we refine the mesh on the remaining sampling region and perform the LSM again in this fine level to label and remove those fine remote and inner cells; e.g., see Figure 1 (bottom-left) for those remote and inner cells. By doing this labeling and removing technique in a multilevel way, we can reconstruct the profile of $\mathbf{D}$ more accurately. We would like to remark that in many cases the number of trimmed cells could be very large and thus save a lot of computational time, especially when the scatterer is composed of multiple components with the distance between each two being several times larger than the 
sizes or diameters of the two components (see, e.g., Figure 1 (bottom-right)).

Now, we are ready to formulate our algorithm in detail. In the following, the sampling region $\Omega$ is always chosen to be a square in $\mathbb{R}^{2}$. Then, let $\left\{\mathcal{T}_{k}\right\}_{k=1}^{L}$ be a nested sequence of meshes on the sampling domain $\Omega$ such that $\mathcal{T}_{k+1}$ is a refinement of $\mathcal{T}_{k}$ for $k=1, \ldots, L-1$. Throughout, we assume that $\mathcal{T}_{k+1}$ is an $n_{k+1} \times n_{k+1}$ mesh while $\mathcal{T}_{k}$ is an $n_{k} \times n_{k}$ mesh, where $n_{k+1}=2 n_{k}-1$ for $k=1, \ldots, L-1$. That is, we refine the mesh $\mathcal{T}_{k}$ by equally subdividing every subsquare in $\mathcal{T}_{k}$ into four subsquares of $\mathcal{T}_{k+1}$. Then if the mesh length of $\mathcal{T}_{k}$ is $h_{k}$ for $k=1, \ldots, L-1$, then $h_{k+1}=h_{k} / 2$. Now, the MLSM can be formulated as follows.

\section{Algorithm MLSM.}

1. Set $k=0$ and choose an initial mesh for the sampling region $\Omega$.

2. Apply the LSM scheme on the $k$ th-level mesh to investigate those mesh points which have not been examined previously.

3. For a given cut-off value $c$, independent of the level $k$, classify and label the $k$ th-level subsquares (cells) into three sets - namely, remote cells, boundary cells, and inner cells - based on the cut-off value principle in the LSM. A cell is labeled as "remote" if the norms of the indicator functions at the vertices of the cell are all larger than $c$, while a cell is labeled as "inner" if the norms of the indicator functions at the vertices of the cell are all less than or equal to $c$, and other remaining cells will be labeled as "boundary cells." Then remove the remote and inner cells.

4. Refine the remaining sampling mesh.

5. Set $k=k+1$, and if $k \leq L$, go to Step 2 .

It is remarked that in order to exclude the extreme case that the obstacle is trapped into a single subsquare of the sampling mesh, the initial mesh should be chosen to be mildly fine such that both "remote" cells and "inner" cells exist.

Next, we will show that the MLSM algorithm is asymptotically optimal in computational complexity. For this purpose, we first present some lemmas. In the following, we denote by $\Gamma$ a $C^{2}$-smooth curve in $\mathbb{R}^{2}$ which forms the boundary of a bounded domain G. For any $h>0$, we define two curves parallel to $\Gamma$ :

$$
\begin{aligned}
\Gamma_{h}^{+}:=\{x+h \nu(x), & x \in \Gamma, \text { and } \nu(x) \text { is the unit normal to } \Gamma \text { at } x \\
& \text { directed to the exterior of } \mathbf{G}\}, \\
\Gamma_{h}^{-}:=\{x-h \nu(x), & x \in \Gamma, \text { and } \nu(x) \text { is the unit normal to } \Gamma \text { at } x \\
& \text { directed to the exterior of } \mathbf{G}\} .
\end{aligned}
$$

Then we have the following lemma.

Lemma 2.1. There exist constants $h_{0}^{+}>0$ and $0<\alpha_{0}^{+} \leq 1$ such that

$$
\operatorname{dist}\left(\Gamma, \Gamma_{h}^{+}\right) \geq \alpha_{0}^{+} h \quad \text { whenever } 0<h<h_{0}^{+} .
$$

Proof. Assume contrarily that there are no constants $h_{0}^{+}$and $\alpha_{0}^{+}$such that (2.3) holds. Then, for $\hat{h}_{1}=1 / 2$, there must exist an $h_{1}$ such that $0<h_{1}<\hat{h}_{1}$ and $\operatorname{dist}\left(\Gamma, \Gamma_{h_{1}}^{+}\right)<h_{1} / 2$; otherwise Lemma 2.1 is true with $h_{0}^{+}=\hat{h}_{1}$ and $\alpha_{0}^{+}=1 / 2$. Next, for $\hat{h}_{2}=\min \left\{h_{1}, 1 / 2^{2}\right\}$, there must exist an $h_{2}$ such that $0<h_{2}<\hat{h}_{2}$ and $\operatorname{dist}\left(\Gamma, \Gamma_{h_{2}}^{+}\right)<h_{2} / 2^{2}$; otherwise Lemma 2.1 is true with $h_{0}^{+}=\hat{h}_{2}$ and $\alpha_{0}^{+}=1 / 2^{2}$. Continuing with this procedure, we have by induction that for $\hat{h}_{k}=\min \left\{h_{k-1}, 1 / 2^{k}\right\}(k \geq$ $3)$, there exists an $h_{k}$ such that $0<h_{k}<\hat{h}_{k}$ and $\operatorname{dist}\left(\Gamma, \Gamma_{h_{k}}^{+}\right)<h_{k} / 2^{k}$. So we obtain 
a positive sequence $\left\{h_{k}\right\}_{k=1}^{\infty}$ such that

$$
\lim _{k \rightarrow \infty} h_{k}=0 \quad \text { and } \quad \lim _{k \rightarrow \infty} \frac{\operatorname{dist}\left(\Gamma, \Gamma_{h_{k}}^{+}\right)}{h_{k}}=0 .
$$

Since both $\Gamma$ and $\Gamma_{h_{k}}^{+}$are compact sets in $\mathbb{R}^{2}$, there exist $x_{k} \in \Gamma$ and $y_{k}^{+} \in \Gamma_{h_{k}}^{+}$for any $k \in \mathbb{N}$ such that

$$
\operatorname{dist}\left(\Gamma, \Gamma_{h_{k}}^{+}\right)=\left|x_{k}-y_{k}^{+}\right|
$$

Set

$$
y_{k}=y_{k}^{+}-h_{k} \nu\left(y_{k}^{+}\right) \in \Gamma \quad \text { for } k \in \mathbb{N},
$$

where $\nu\left(y_{k}^{+}\right)$is the unit outward normal to $\Gamma_{h_{k}}^{+}$at $y_{k}^{+}$. By extracting subsequences if necessary, we may assume that

$$
\lim _{k \rightarrow \infty} x_{k}=x_{0} \quad \text { and } \quad \lim _{k \rightarrow \infty} y_{k}=y_{0} .
$$

By (2.6), (2.7), we see that $\lim _{k \rightarrow \infty} y_{k}^{+}=y_{0}$, which together with (2.4), (2.5) implies that

$$
x_{0}=y_{0}=x^{*}
$$

for some $x^{*} \in \Gamma$. Noting that $\nu(x)$ is continuous, for an arbitrary $\varepsilon>0$ there exists $\delta>0$ such that

$$
\left|\nu(x)-\nu\left(x^{*}\right)\right|<\varepsilon \quad \forall x \in B_{\delta}\left(x^{*}\right) \cap \Gamma,
$$

where $B_{\delta}\left(x^{*}\right)=\left\{x \in \mathbb{R}^{2} ;\left|x-x^{*}\right|<\delta\right\}$. By (2.7) and (2.8), we know that there exists $k_{\varepsilon} \in \mathbb{N}$ such that

$$
x_{k} \in B_{\delta}\left(x^{*}\right), \quad y_{k} \in B_{\delta}\left(x^{*}\right) \quad \forall k>k_{\varepsilon} .
$$

Furthermore, by (2.4), we can assume that $k_{\varepsilon}$ is chosen such that

$$
\frac{\operatorname{dist}\left(\Gamma, \Gamma_{h_{k}}^{+}\right)}{h_{k}}<\frac{1}{2} \quad \forall k>k_{\varepsilon}
$$

namely

$$
\left|x_{k}-y_{k}^{+}\right|<\frac{1}{2} h_{k} \quad \forall k>k_{\varepsilon} .
$$

It is noted that by $(2.11)$ we must have $x_{k} \neq y_{k}$ for all $k>k_{\varepsilon}$, since otherwise we would have $\left|x_{k}-y_{k}^{+}\right|=\left|y_{k}-y_{k}^{+}\right|=h_{k}$. Let $\tau(x)$ be the tangential to $\Gamma$ at $x$, and we know from (2.9) that

$$
\left|\tau(x)-\tau\left(x^{*}\right)\right|<\varepsilon \quad \forall x \in B_{\delta}\left(x^{*}\right) \cap \Gamma .
$$

Next, we investigate the angle $\angle\left(\overrightarrow{x_{k} y_{k}}, \tau\left(y_{k}\right)\right)(\in[0, \pi / 2])$ between the two vectors $\overrightarrow{x_{k} y_{k}}$ and $\tau\left(y_{k}\right)$ for $k>k_{\epsilon}$. From the geometric interpretation of Lagrange's theorem, we 
know that there exists $\xi_{k} \in B_{\delta}\left(x^{*}\right) \cap \Gamma$ such that $\tau\left(\xi_{k}\right)$ is parallel to $\overrightarrow{x_{k} y_{k}}$. By (2.12), we know that

$$
\lambda_{k}:=\left\langle\tau\left(\xi_{k}\right), \tau\left(y_{k}\right)\right\rangle=\left\langle\tau\left(x^{*}\right)-\mathcal{O}(\varepsilon), \tau\left(x^{*}\right)-\mathcal{O}(\varepsilon)\right\rangle=1-\mathcal{O}(\varepsilon) \quad \text { as } \varepsilon \rightarrow+0
$$

where $\langle\cdot, \cdot\rangle$ is the inner product in $\mathbb{R}^{2}$. Hence,

$$
\theta_{k}:=\angle\left(\overrightarrow{x_{k} y_{k}}, \tau\left(y_{k}\right)\right)=\arccos \lambda_{k}=\mathcal{O}(\sqrt{\varepsilon}) \quad \text { as } \varepsilon \rightarrow+0 .
$$

Now, let $\triangle x_{k} y_{k} y_{k}^{+}$denote the triangle with vertices $x_{k}, y_{k}$, and $y_{k}^{+}$. It is easily seen that the interior angle of $\triangle x_{k} y_{k} y_{k}^{+}$at $y_{k}$, namely $\angle\left(\overrightarrow{x_{k} y_{k}}, \overrightarrow{y_{k}^{+} y_{k}}\right)$, is either $\pi / 2+\theta_{k}$ or $\pi / 2-\theta_{k}$. Then, by (2.10) and (2.14), we take $\varepsilon_{0}>0$ to be sufficiently small and $k_{\varepsilon_{0}} \in \mathbb{N}$ be sufficiently large such that for all $k>k_{\varepsilon_{0}}$,

$$
\frac{\left|x_{k}-y_{k}^{+}\right|}{h_{k}}<\frac{1}{2} \quad \text { and } \quad \sin \left(\frac{\pi}{2}-\theta_{k}\right)>\frac{1}{2}
$$

Then, in the case that $\angle\left(\overrightarrow{x_{k} y_{k}}, \overrightarrow{y_{k}^{+} y_{k}}\right)=\pi / 2+\theta_{k}>\pi / 2$,

$$
\left|x_{k}-y_{k}^{+}\right|>\left|y_{k}-y_{k}^{+}\right|=h_{k},
$$

and in the case that $\angle\left(\overrightarrow{x_{k} y_{k}}, \overrightarrow{y_{k}^{+} y_{k}}\right)=\pi / 2-\theta_{k}$,

$$
\frac{\left|x_{k}-y_{k}^{+}\right|}{h_{k}}=\frac{\left|x_{k}-y_{k}^{+}\right|}{\left|y_{k}-y_{k}^{+}\right|} \geq \sin \left(\pi / 2-\theta_{k}\right)>\frac{1}{2} \text {. }
$$

In both cases, we have a contradiction with the first inequality in (2.15). This completes the proof of Lemma 2.1.

Lemma 2.2. There exist constants $h_{0}^{-}>0$ and $0<\alpha_{0}^{-} \leq 1$ such that

$$
\operatorname{dist}\left(\Gamma, \Gamma_{h}^{-}\right) \geq \alpha_{0}^{-} h \quad \text { whenever } 0<h<h_{0}^{-} .
$$

Proof. The lemma can be proved in a most similar way to that of Lemma 2.1.

LEMma 2.3. There exist constants $h_{0}>0$ and $\alpha_{0}>0$ such that

$$
\operatorname{dist}\left(\Gamma, \Gamma_{\alpha_{0} h}^{ \pm}\right) \geq \sqrt{2} h \quad \text { whenever } 0<h<h_{0}
$$

Proof. Set

$$
\alpha_{0}=\frac{\sqrt{2}}{\min \left(\alpha_{0}^{+}, \alpha_{0}^{-}\right)} \quad \text { and } \quad h_{0}=\frac{\min \left(h_{0}^{+}, h_{0}^{-}\right)}{\alpha_{0}},
$$

where $\alpha_{0}^{ \pm}$and $h_{0}^{ \pm}$are constants given in Lemmas 2.1 and 2.2. Then, it is easy to verify that when $h<h_{0}$, namely $\alpha_{0} h<\min \left(h_{0}^{+}, h_{0}^{-}\right)$,

$$
\operatorname{dist}\left(\Gamma, \Gamma_{\alpha_{0} h}^{+}\right) \geq \alpha_{0}^{+} \alpha_{0} h \geq \sqrt{2} h
$$

and

$$
\operatorname{dist}\left(\Gamma, \Gamma_{\alpha_{0} h}^{-}\right) \geq \alpha_{0}^{-} \alpha_{0} h \geq \sqrt{2} h .
$$

Copyright ( by SIAM. Unauthorized reproduction of this article is prohibited. 
The following theorem is crucial to our subsequent investigation.

THEOREM 2.4. Let $\mathcal{T}$ be an $n \times n$ mesh on the sampling region $\Omega$. There exist two constants $\kappa_{0}>0$ and $n_{0} \in \mathbb{N}$ such that $\partial \mathbf{D}$ lies on at most $\kappa_{0} n$ subsquares of $\mathcal{T}$ for all $n \geq n_{0}$.

Proof. To ease the discussion, we assume that the scatterer $\mathbf{D}$ is composed of a single component obstacle. That is, $\mathbf{D}$ is a bounded domain. But we remark that our subsequent proof can be easily modified to the case that $\mathbf{D}$ has finitely many connected components.

Let $\Gamma:=\partial \mathbf{D}$ in Lemma 2.3. Take $n \in \mathbb{N}$ to be sufficiently large such that the mesh length $h$ of $\mathcal{T}$ satisfies $h<h_{0}$. Suppose that $\partial \mathbf{D}$ lies on $m$ subsquares of $\mathcal{T}$. By (2.17) and (2.18), it is easily seen that these $m$ subsquares must lie in the ring-shaped region formed by $\Gamma_{\alpha_{0} h}^{+}$and $\Gamma_{\alpha_{0} h}^{-}$. Let $s_{0}$ denote the area occupied by this ring-shaped region, $\omega_{0}=|\Omega|$ be the area of $\Omega$, and $\eta_{0}=|\partial \mathbf{D}|$ be the length of the boundary curve $\partial \mathbf{D}$. Then, we have

$$
m h^{2} \leq s_{0} \leq 2 \eta_{0} \alpha_{0} h
$$

hence

$$
m \leq \frac{2 \eta_{0} \alpha_{0}}{h}
$$

By noting $n^{2} h^{2}=\omega_{0}$, we further have

$$
m \leq \frac{2 \eta_{0} \alpha_{0}}{\sqrt{\omega_{0}}} n
$$

Now, the theorem is seen to be held with

$$
\kappa_{0}=\left\lceil\frac{2 \eta_{0} \alpha_{0}}{\sqrt{\omega_{0}}}\right\rceil
$$

where for a positive number $a,\lceil a\rceil$ denotes the smallest integer not less than $a$.

The above theorem shows that for a sufficient fine $n \times n$ mesh, $\partial \mathbf{D}$ lies on at most $\mathcal{O}(n)$ subsquares. We next show that $\partial \mathbf{D}$ also lies on at least $\mathcal{O}(n)$ subsquares.

THEOREM 2.5. Let $\mathcal{T}$ be an $n \times n$ mesh on the sampling region $\Omega$. There exist two constants $\beta_{0}>0$ and $m_{0} \in \mathbb{N}$ such that $\partial \mathbf{D}$ lies on at least $\beta_{0} n$ subsquares of $\mathcal{T}$ for all $n \geq m_{0}$.

Proof. As in Theorem 2.4, we need only to consider the simple case that $\mathbf{D}$ is a connected bounded domain, and that the subsequent proof is easily modified to the case that $\mathbf{D}$ has finitely many connected components.

By our assumption on the sampling mesh, we may choose $\mathcal{T}$ to be fine enough such that there is at least one inner cell. Take one of the edges of this cell and denote its connected extension in $\mathbf{D}$ by $A B$ with the two endpoints $A$ and $B$ lying on $\partial \mathbf{D}$ (see Figure 2). We suppose that $A B$ lies on $m$ subsquares of $\mathcal{T}$. Let $A_{0}, A_{1}, \ldots, A_{m}$ be the vertices of those subsquares, all lying on the extended line of $A B$ and ordered in the direction from $A$ to $B$ (see Figure 2). By our organization, $A$ either is $A_{0}$ or lies between $A_{0}$ and $A_{1}$, and $B$ either is $A_{m}$ or lies between $A_{m-1}$ and $A_{m}$, whereas $A_{j}, j=$ $1,2, \ldots, m-1$, all lie inside of $\mathbf{D}$. We denote by $l_{0}, l_{1}, \ldots, l_{m}$ those line segments of $\mathcal{T}$ in $\Omega$ which, respectively, pass through $A_{0}, A_{1}, \ldots, A_{m}$. Noting that $\mathbf{D}$ is connected, by the fundamental property of connected set, we know that $l_{j}, j=1,2, \ldots, m-1$ must have intersection with $\partial \mathbf{D}$. We denote by $A_{1}^{\prime}{ }_{1}, A_{2}^{\prime}, \ldots, A_{m-1}^{\prime}$ those intersection 


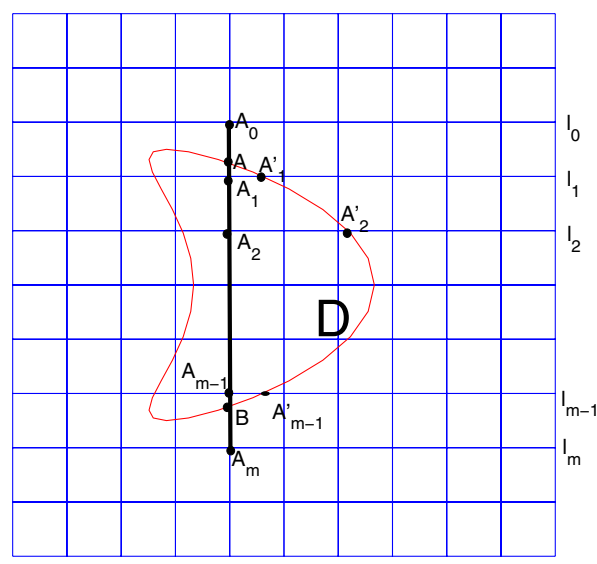

FIG. 2. Illustration of the proof of Theorem 2.5.

points which lie on one side of $A B$. It is remarked that $A_{j}$ for $j=1, \ldots, m-1$ is not necessarily unique. Now, by the connectedness of $\partial \mathbf{D}$, we know that between $A$ and $A^{\prime}{ }_{1}, B$ and $A^{\prime}{ }_{m-1}$, and $A^{\prime}{ }_{j}$ and $A^{\prime}{ }_{j+1}$ for $j=1, \ldots, m-2$ there must be a connected part of $\partial \mathbf{D}$ which lies in the stripped region, respectively formed by $l_{0}$ and $l_{1}, l_{m-1}$ and $l_{m}$, and $l_{j}$ and $l_{j+1}$ for $j=1, \ldots, m-2$. Therefore, if we suppose that $\partial \mathbf{D}$ lies on $m^{\prime}$ subsquares of $\mathcal{T}$, then there must be at least one from those subsquares which lies in the stripped region formed by $l_{j}$ and $l_{j+1}$ for $j=0,1, \ldots, m-1$. Hence, we have $m^{\prime} \leq m$. Next, we set $A_{0}=A$ and $A_{m}=B$, and by noting that $\left|A_{j} A_{j+1}\right| \leq h$ for $j=0,1, \ldots, m-1$, we have

$$
\sum_{j=0}^{m-1}\left|A_{j} A_{j+1}\right| \leq m h, \quad \text { i.e., }|A B| \leq m h .
$$

Finally, we have by noting $n^{2} h^{2}=|\Omega|$ that

$$
m^{\prime} \geq m \geq|A B| \frac{1}{h} \geq \beta_{0} n,
$$

with $\beta_{0}=|A B| / \sqrt{|\Omega|}$. This completes the theorem.

Theorems 2.4 and 2.5 reveal that in order to achieve a good reconstruction of the scatterer $\mathbf{D}$, we need to at least solve $\mathcal{O}(n)$ far-field equations (1.4) with a fine $n \times n$ sampling mesh. Now, we are ready to present the main result - that the algorithm MLSM possesses the asymptotically optimal computational complexity.

THEOREM 2.6. Consider an L-level MLSM algorithm with a nested sequence of sampling mesh $\left\{\mathcal{T}_{k}\right\}_{k=1}^{L}$. Suppose that for each $k, \mathcal{T}_{k}$ is of size $n_{k} \times n_{k}$ with mesh length $h_{k}$ such that $0<h_{1}<h_{0}$, where $h_{0}$ is given in Lemma 2.3 corresponding to $\partial \mathbf{D}$. Then, by using the MLSM to reconstruct $\partial \mathbf{D}$, the far-field equation (1.4) is solved $\mathcal{O}\left(n_{L}\right)$ times in total.

Proof. We denote by $\mathcal{C}_{k}, k=1,2, \ldots, L$, the points to be investigated on the $k$ th level. By Theorem 2.4, we know that $\partial \mathbf{D}$ lies on at most $\kappa_{0} n_{k}$ subsquares of $\mathcal{T}_{k}$. Next, when we turn to the $(k+1)$ th level, by our description of the MLSM, we need 


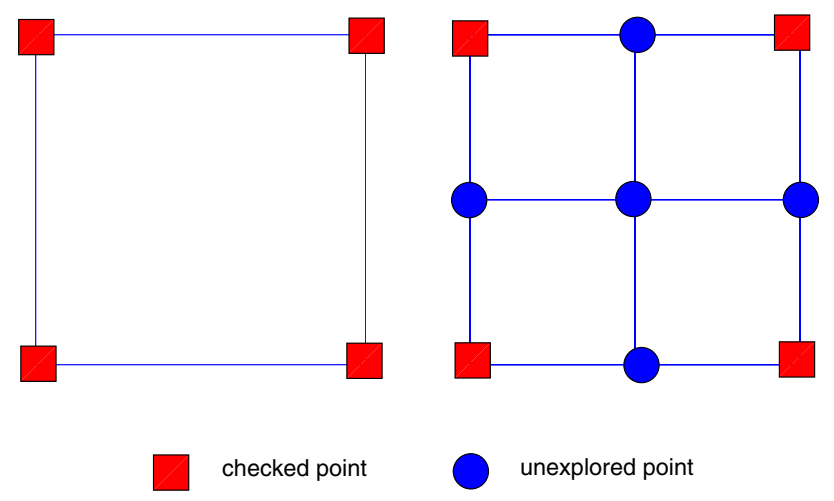

FIG. 3. Illustration of the proof of Theorem 2.6.

only to investigate the mesh points on those subsquares of $\mathcal{T}_{k+1}$ which have not been examined before and can be easily seen to be at most $5 \kappa_{0} n_{k}$ mesh points as shown in Figure 3. Hence, we have

$$
\mathcal{C}_{k} \leq \mathcal{C}_{k-1}+5 \kappa_{0} n_{k-1}, \quad k=2, \ldots, L,
$$

where $n_{k-1}=\left(n_{k}+1\right) / 2$. Recursively, we can obtain

$$
\begin{aligned}
\mathcal{C}_{L} & \leq \mathcal{C}_{L-1}+5 \kappa_{0} n_{L-1}, \\
\mathcal{C}_{L-1} & \leq \mathcal{C}_{L-2}+5 \kappa_{0} n_{L-2}, \\
& \ldots \ldots \\
\mathcal{C}_{2} & \leq \mathcal{C}_{1}+5 \kappa_{0} n_{1} .
\end{aligned}
$$

By summing up the above inequalities we get

$$
\mathcal{C}_{L} \leq \mathcal{C}_{1}+5 \kappa_{0}\left[n_{L-1}+n_{L-2}+\cdots+n_{1}\right] .
$$

Since it is easy to deduce that $n_{L-k}=n_{L} / 2^{k}+\sum_{j=1}^{k} 1 / 2^{j}$ for $k=1,2, \ldots, L-1$, we see that

$$
\mathcal{C}_{L} \leq \mathcal{C}_{1}+5 \kappa_{0}\left(L+n_{L}\right)
$$

i.e.,

$$
\mathcal{C}_{L} \leq \mathcal{O}\left(n_{L}\right) \quad \text { for sufficiently large } n_{L} \in \mathbb{N} .
$$

This means that the MLSM has the asymptotically optimal computational complexity. This completes the proof.

Remark 2.7. As we have pointed out earlier, all of the results in this section can be modified to the $\mathbb{R}^{3}$ case, where the MLSM algorithm needs to solve far-field equations (1.4) $\mathcal{O}\left(n_{L}^{2}\right)$ times. 
3. Numerical experiments with discussions. In this section, we perform three tests to illustrate the effectiveness and efficiency of the newly proposed MLSM algorithm. All of the programs in our experiments are written in MATLAB and run on a Pentium $3 \mathrm{GHz}$ PC.

The scatterer in system (1.1) will be chosen to be the kite-shaped object which has been widely tested in inverse scattering problems (see, e.g., [5], [8], and [12]). There are a total of three tests to be considered, which are, respectively, referred to as SK, SKn, and DKn. For experiments SK and SKn, the scatterer D is composed of a single kite. However, in experiment SK, we would not add noise to the synthetic far-field data, and in experiment SKn, we add random noise. For experiment DKn, the scatterer $\mathbf{D}$ is composed of two kites, and the synthetic far-field data is also added with random noise. The other parameters chosen for these experiments are listed in Table 1.

TABLE 1

Experimental parameters for the tests.

\begin{tabular}{cccc}
\hline \hline & Test $1(\mathrm{SK})$ & Test 2 (SKn) & Test 3 (DKn) \\
\hline Sampling domain $\Omega$ & {$[-3,3] \times[-3,3]$} & {$[-3,3] \times[-3,3]$} & {$[-4,8] \times[-4,8]$} \\
Incident wave number $k$ & 1 & 1 & 1 \\
Finest level $n_{L}$ & 129 & 129 & 129 \\
Upper threshold $c_{1}$ & 0.03 & 0.032 & 0.03 \\
Lower threshold $c_{2}$ & 0.03 & 0.032 & 0.02 \\
Noise level $\delta$ & 0 & 0.10 & 0.05 \\
\hline No. of incident directions & \multicolumn{4}{|}{} \\
No. of observation directions & \multicolumn{3}{|}{} \\
\hline \hline
\end{tabular}

It is noted that for experiment DKn, we have taken two cut-off values, $c_{1}$ and $c_{2}$, $c_{1}<c_{2}$, instead of only one cut-off value, $c$. Since in DKn, the scatterer is composed of two kites, it is better to take a range of cut-off values, i.e., $\left[c_{1}, c_{2}\right]$, which enables us to get a buffer region of locating the boundary of the underlying object. Like in the original LSM, we label as inner those points with the norm of distributed density $g$ less than $c_{1}$ and remote those points with the norm of distributed density $g$ greater than $c_{2}$.

The synthetic far-field data are generated by solving the layer potential operator equation with Nyström's method (see section 3.5, Chapter 3 in [7]). We compute the far-field patterns at 32 equidistantly distributed observation points $\left(\cos t_{j}, \sin t_{j}\right)$, $t_{j}=2 j \pi / 32, j=0,1, \ldots, 31$, and 32 equidistantly distributed incident directions $\left(\cos \tau_{j}, \sin \tau_{j}\right), \tau_{j}=2 j \pi / 32, j=0,1, \ldots, 31$. The far-field patterns we obtain are subjected pointwise to uniform random noise. The uniform random noise is added according to the following formula:

$$
u_{\infty}^{\delta}=u_{\infty}+\delta r_{1}\left|u_{\infty}\right| \exp \left(i \pi r_{2}\right),
$$

where $r_{1}$ and $r_{2}$ are two uniform random numbers, both ranging from -1 to 1 , and $\delta$ is the noise level. For each mesh point $z$, the corresponding far-field equation (1.4) is solved by using the Tikhonov regularization method (cf. [3]), with the regularization parameter determined by the standard L-curve method.

In tests 1 and 2, the kite-shaped object $\mathbf{D}$ is shown in Figure 4 with the boundary $\partial \mathbf{D}$ given by the following parametric form:

$$
x(t)=(\cos t+0.65 \cos 2 t-0.65,1.5 \sin t), \quad 0 \leq t \leq 2 \pi .
$$




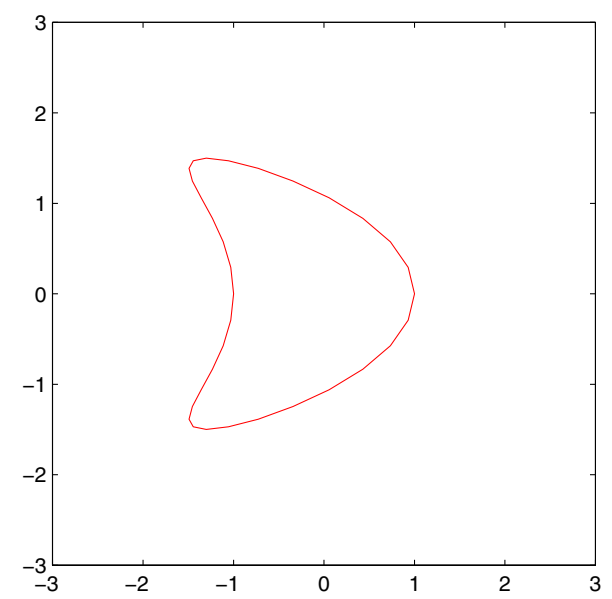

FIG. 4. Kite-shaped obstacle.

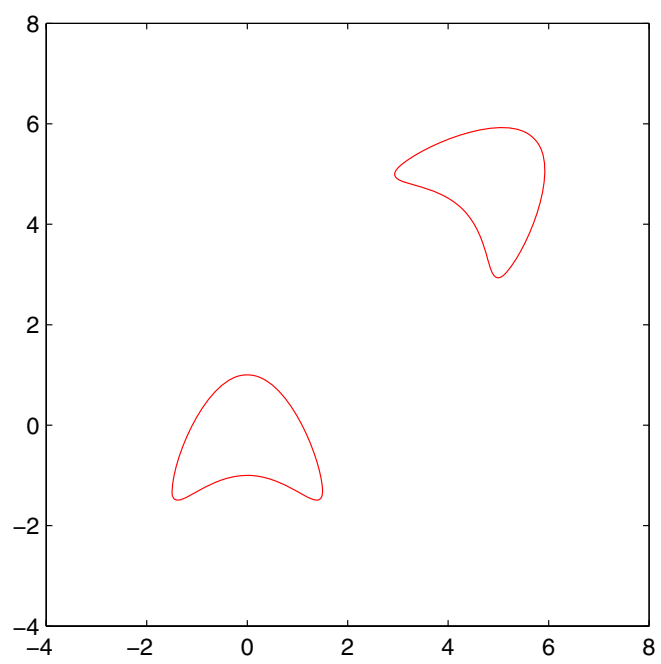

FIG. 5. Two kite-shaped objects.

For test 3, the two kite-shaped objects are shown in Figure 5, which are derived from the kite in Figure 4 by rigid motions: the bottom-left one is given by the one in Figure 4 after a counterclockwise $\pi / 2$ rotation, and the top-right one is given by the one in Figure 4 after a counterclockwise $\pi / 4$ rotation and 5-unit displacement in both longitude and latitude directions.

We now turn to experiment SK. First, we solve the far-field equation (1.4) on the finest mesh $(129 \times 129)$ to find $g^{z}$ with $z$ being a sampling mesh point. In order to have a view of the behavior of this $g^{z}$ over the sampling mesh, we plot the negative logarithm of its $L^{2}$-norm, namely - $\log \left\|g^{z}\right\|_{L^{2}\left(\mathbb{S}^{1}\right)}$, in a 3D graph (see Figure 6(a)), but 


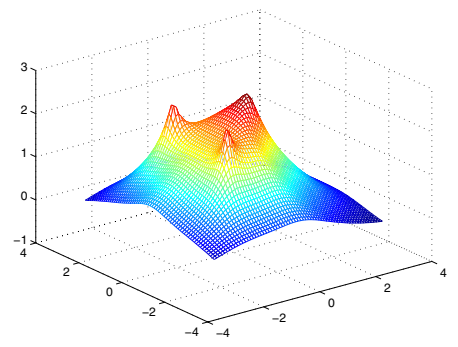

(a) The negative logarithm of the $L^{2}$-norm of $g^{z}$ plotted in 3D.

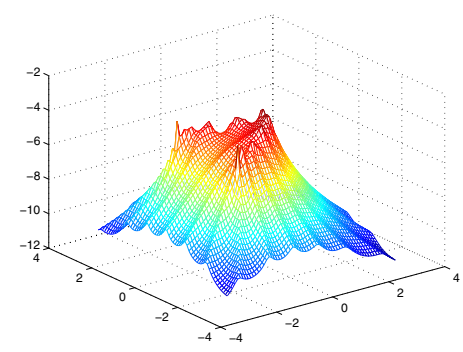

(c) The negative logarithm of the $L^{2}$-norm of $g^{z}$ plotted in 3D without regularization in deriving $g^{z}$.

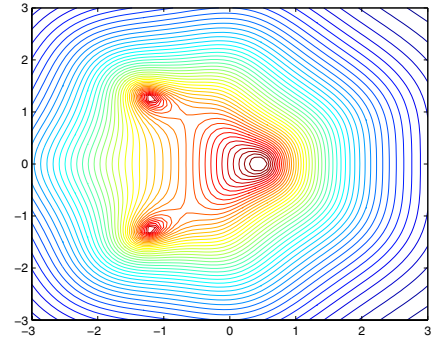

(b) Contours of the negative logarithm of the $L^{2}$-norm of $g^{z}$.

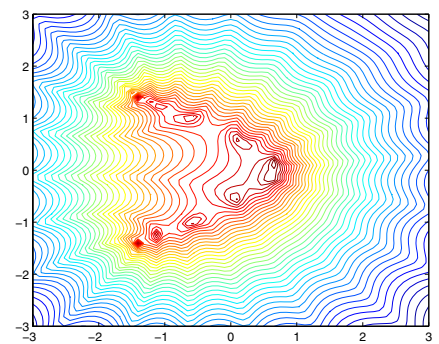

(d) Contours of the negative logarithm of the $L^{2}$-norm of $g^{z}$ without regularization in deriving $g^{z}$.

FIG. 6. Test 1 (SK): Surface and contours of the negative logarithm of the $L^{2}$-norm of $g^{z}$ with and without regularization.

such scalings are not needed in our MLSM procedure for those tests. The corresponding contours for $-\log \left\|g^{z}\right\|_{L^{2}\left(\mathbb{S}^{1}\right)}$ are also given in Figure 6(b) for a 2D view. Then, we can use the cut-off value principle to detect the kite, and this gives the original LSM. In this case, we would like to refer the reader to [9] for a glance at the numerical outcome. We remark that the regularization is crucial in the numerical procedure. Even in this noise-free case with $\delta=0$, regularization is still necessary since the exact far-field data $u_{\infty}$ is not available and computed here numerically by using Nyström's method, thus causing some approximation errors in addition to the normal roundingoff errors. We have also plotted the negative logarithm of the $L^{2}$-norm of $g^{z}$ obtained by solving the far-field equation without regularization, from which it can be seen that the reconstruction would be rather unsatisfactory; see the 3D display and $2 \mathrm{D}$ contour curves in Figure 6(c) and Figure 6(d), respectively. This phenomenon reflects the ill-posed nature of the problem at hand and is consistent with the one observed in [9].

Next, we apply our (6-level) MLSM to this problem with $n_{L}:=n_{6}=129$ and plot the evolution of the detected boundary of the underlying object level-by-level. Figures 7 and 8 demonstrate that the boundary of the kite-shaped object can be approximated in a clearly improving manner as we go from coarse to fine meshes, but the points 

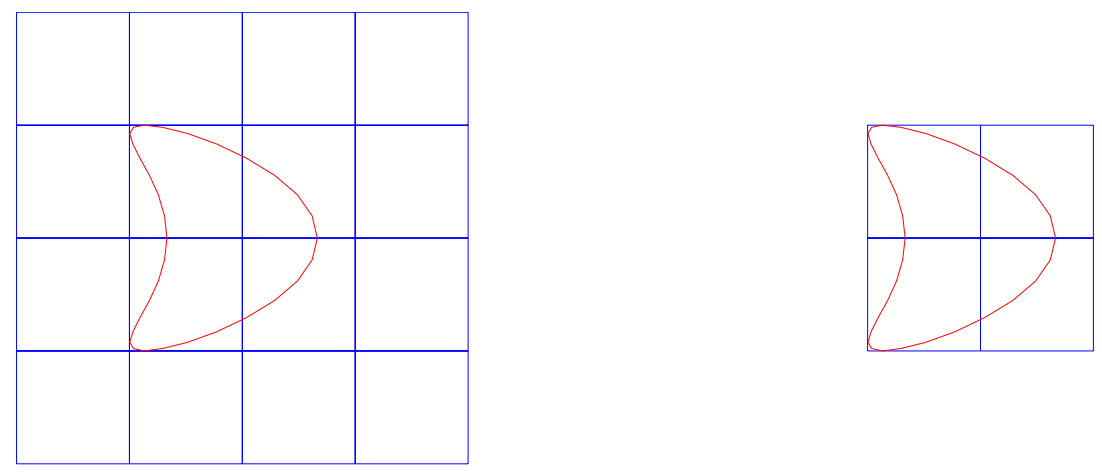

level 1
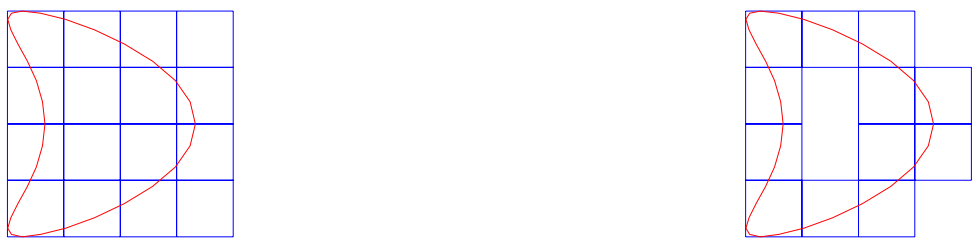

level 2
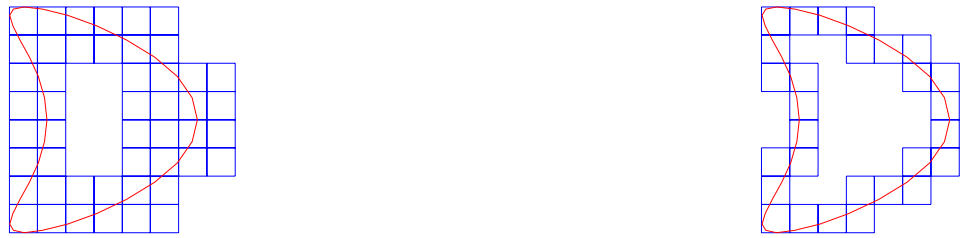

level 3

FIG. 7. MLSM iteration for test 1 (SK). Figures on the left: Refinement of the previous coarse grid. Figures on the right: The remote and inner cells are removed.

Copyright (c) by SIAM. Unauthorized reproduction of this article is prohibited. 

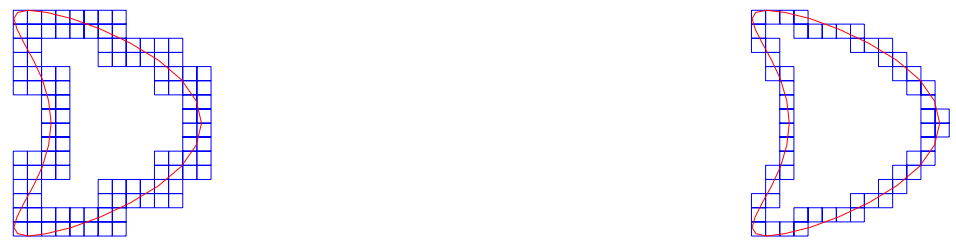

level 4
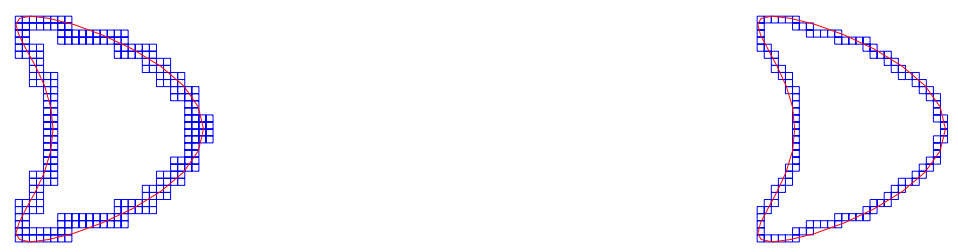

level 5
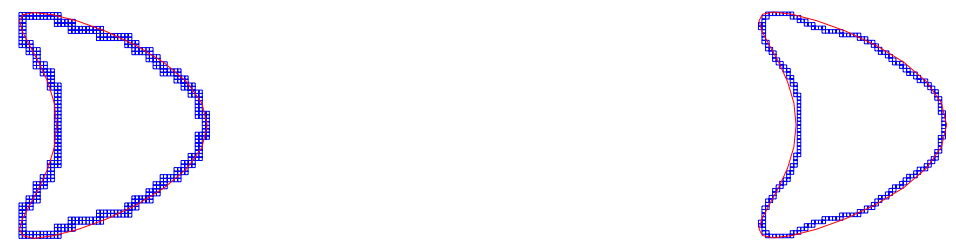

level 6

FIG. 8. MLSM iteration for test 1 (SK). Figures on the left: Refinement of the previous coarse grid. Figures on the right: The remote and inner cells are removed.

Copyright $@$ by SIAM. Unauthorized reproduction of this article is prohibited. 
TABLE 2

Number of points checked by the MLSM at each level and the total number of points checked by $M L S M$ and LSM in the tests.

\begin{tabular}{ccccccccc}
\hline \hline & \multicolumn{7}{c}{ Level of grid } & \\
\cline { 2 - 6 } & 1 & 2 & 3 & 4 & 5 & 6 & MLSM & LSM \\
\hline Test 1 & 25 & 8 & 25 & 56 & 121 & 248 & 483 & 16641 \\
Test 2 & 25 & 8 & 34 & 61 & 129 & 260 & 517 & 16641 \\
Test 3 & 25 & 24 & 36 & 71 & 163 & 375 & 694 & 16641 \\
\hline
\end{tabular}

examined are kept within the order $O\left(n_{L}\right)$. This first experiment SK suggests that the MLSM performs as well as the original LSM method but the computational costs have reduced significantly. We have also counted the numbers of points which have been exploited in the MLSM and listed them in Table 2. For test 1, it is 483 and this is roughly one thirtieth of that for the LSM, which is $16641(=129 \times 129)$. Here we would like to point out an important observation about the implementation of the MLSM: If at a certain level, a cell is labeled as remote (or inner) and we trim it from this level, but part of its boundary is left for the next level, then all of the sampling points of this fine level lying on that part of the boundary should be labeled as having been exploited, since they are obviously remote (resp., inner). We take the number 8 in the second level for test 1 as an illustration. From the left subfigure of level 2 in Figure 7, we know that a total of 16 new points come out from the refinement of the mesh. But eight of these sixteen points, which lie on the outermost boundary of the second level mesh, need not be exploited by our MLSM. This is because they are on the boundary of some trimmed remote cells from the first level; we know that they are remote points without exploiting. The same rule applies in order to interpret the numbers at the finer levels and the other tests.

Next, we add $10 \%$ uniform random noise to the far-field data and run the MLSM again for the SKn case. The evolution of the boundary of the kite is illustrated in Figures 9 and 10. We see the total number of the points examined to locate the boundary is 517 , almost the same as the previous SK case (see Table 2).

Then, we test our MLSM for the DKn case with $5 \%$ uniform random noise to the far-field data and plot the evolution of the boundary of these two kites in Figures 11 and 12. Note that there is a slight increase in the number of exploited points which is due to a buffer range of cut-off values used in this test.

Finally, we plot all the subsquares that have been checked in the MLSM procedure in a single figure for all of the above experiments; see Figure 13 for test 1 (SK), Figure 14 for test $2(\mathrm{SKn})$, and Figure 15 for test $3(\mathrm{DKn})$. From those figures, we get a have concrete feeling about how MLSM works to identify the boundary of the underlying object.

For comparison, we list in Table 2 the number of points examined at each level in the MLSM procedure and the total number of points examined by MLSM and LSM for all of the three tests. It can be seen from Table 2 that the number of points examined at each level is about $\sigma_{0} n_{k}$ with $\sigma_{0} \approx 2$, which is consistent with our theoretical analysis in section 2 .

To consolidate the asymptotically optimal computational complexity, we perform the three tests again with the mesh size $n_{L}$ in the finest level being $33,65,129$, and 257 , respectively. But for all of those experiments, we start with the coarsest mesh given by $n_{1}=5$. Furthermore, we let the cut-off value $c$ be the average of $c_{1}$ and $c_{2}$ in Table 1, and this is to eliminate the possible deterioration due to the additional 

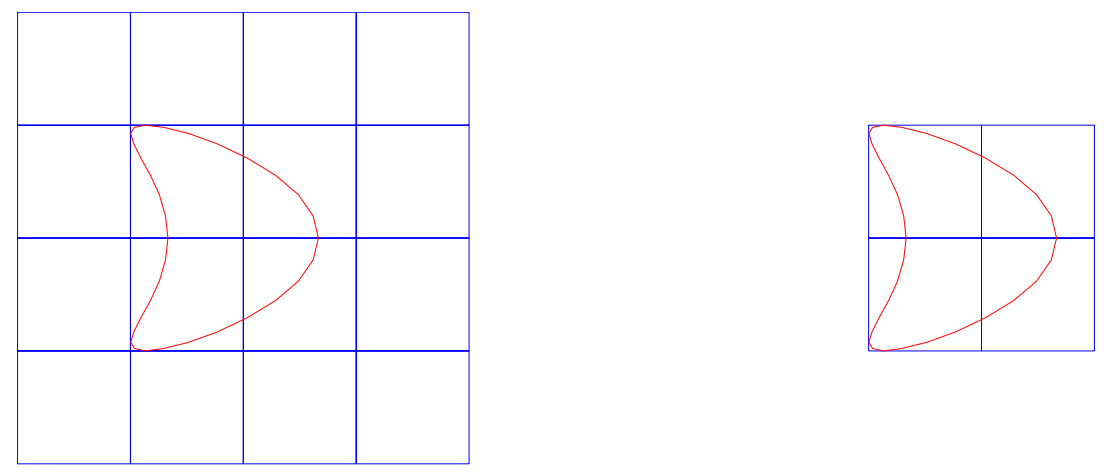

level 1
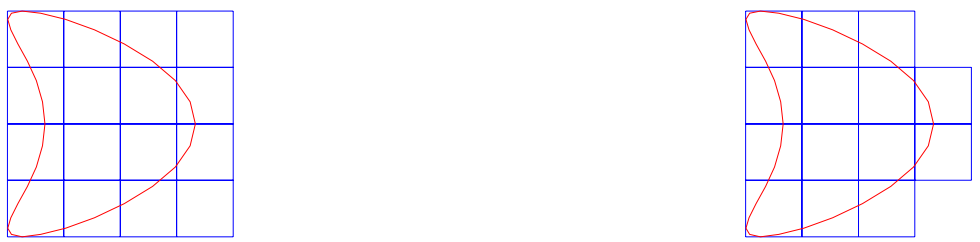

level 2
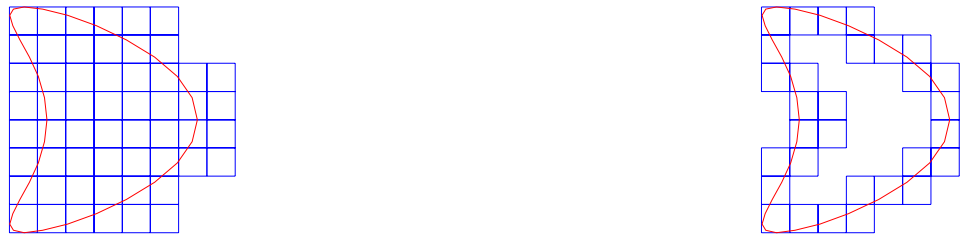

level 3

FIG. 9. MLSM iteration for test 2 (SKn). Figures on the left: Refinement of the previous coarse grid. Figures on the right: The remote and inner cells are removed.

Copyright (C) by SIAM. Unauthorized reproduction of this article is prohibited. 

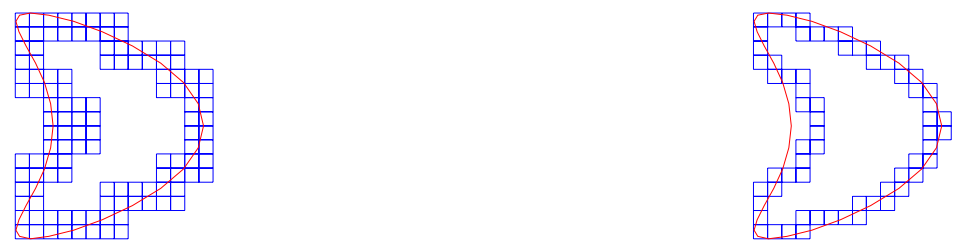

level 4
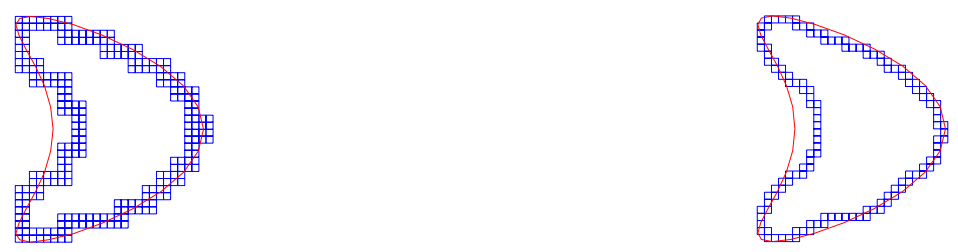

level 5
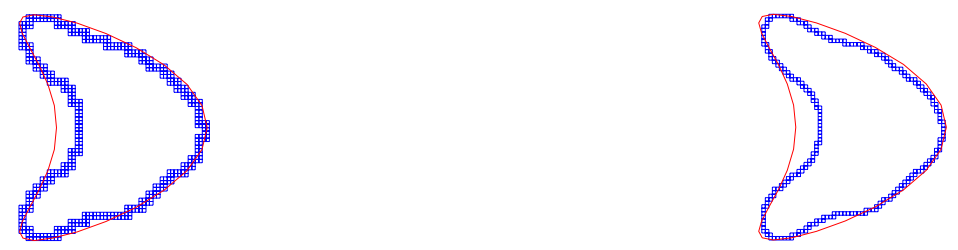

level 6

FIG. 10. MLSM iteration for test 2 (SKn). Figures on the left: Refinement of the previous coarse grid. Figures on the right: The remote and inner cells are removed.

Copyright $@$ by SIAM. Unauthorized reproduction of this article is prohibited. 

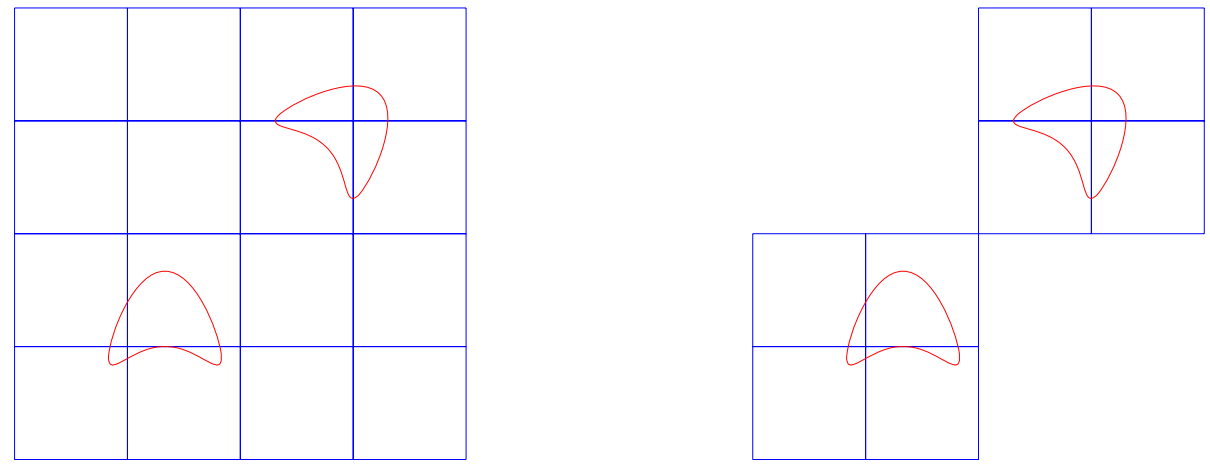

level 1
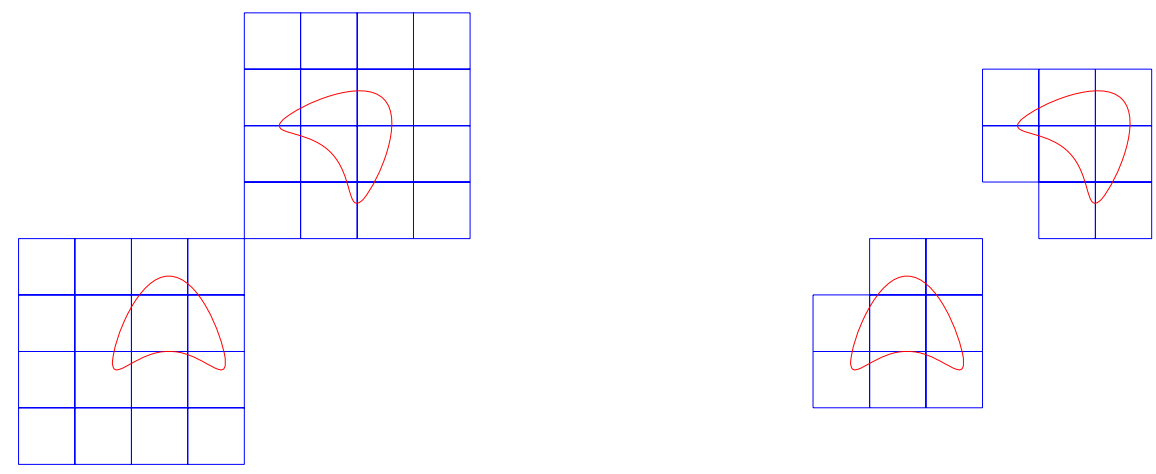

level 2
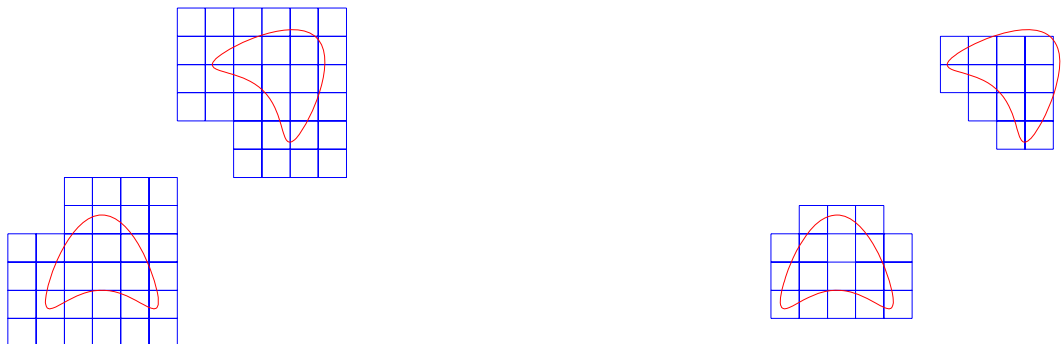

level 3

FIG. 11. MLSM iteration for test 3 (DKn).

Copyright (c) by SIAM. Unauthorized reproduction of this article is prohibited. 

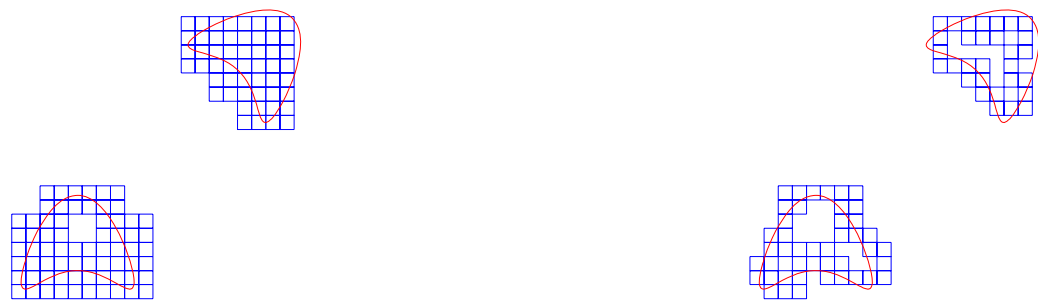

level 4
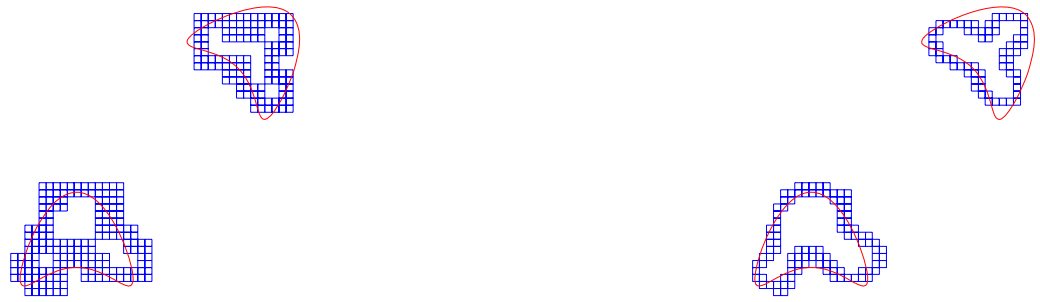

level 5
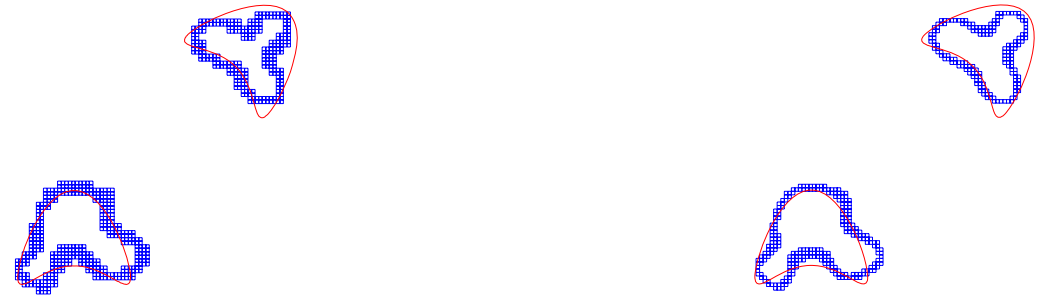

level 6

FIG. 12. MLSM iteration for test 3 (DKn).

Copyright (c) by SIAM. Unauthorized reproduction of this article is prohibited. 


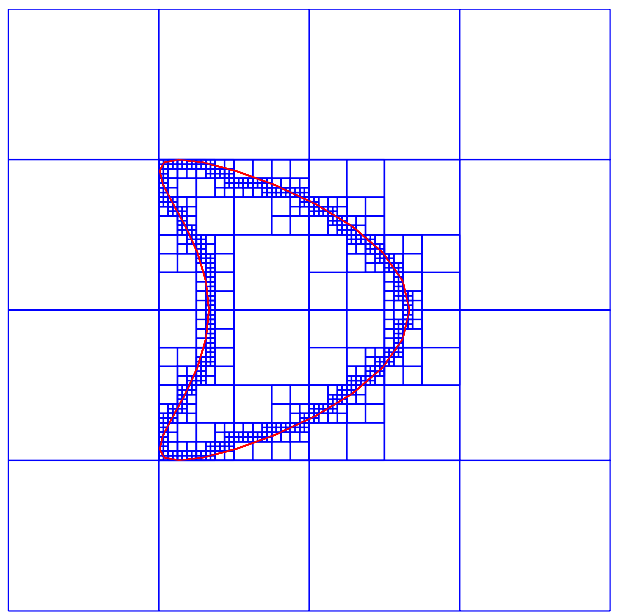

FIG. 13. One kite-shaped object (SK).

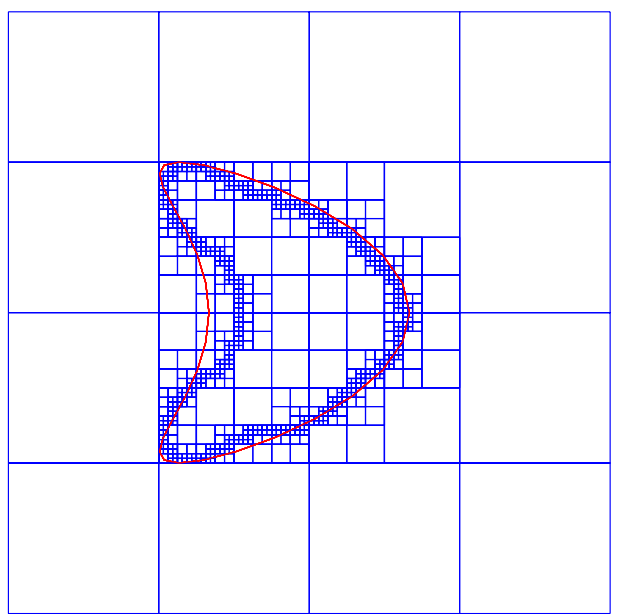

FIG. 14. One kite-shaped object (SKn).

points checked in the buffer region. The total number of points examined and the time for each test are listed in Table 3. Moreover, we compare the time cost between MLSM and LSM only for test 2, since the computational cost for investigating one point is relatively fixed, the time cost for tests 1 and 3 is of slight difference compared with that for test 2 by using the LSM. As shown in Table 3, the computational cost for MLSM grows linearly as $n_{L}$ increases, compared with the quadratic increase of the time consumption of the traditional LSM. It can be seen that the number of far-field 


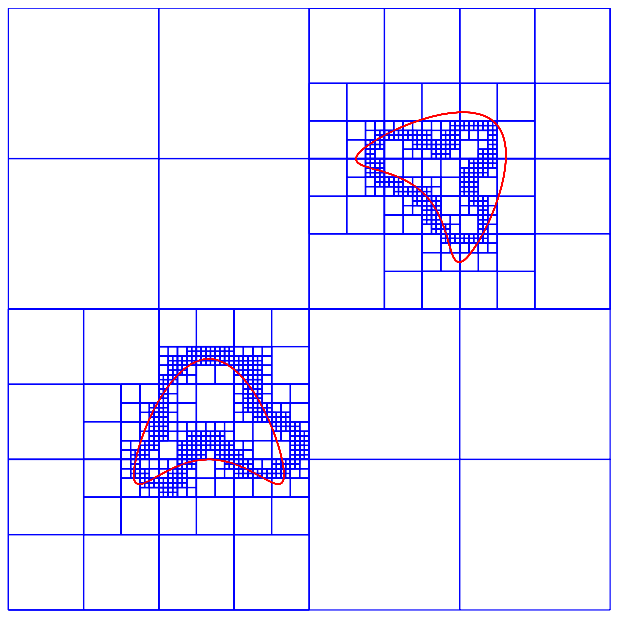

FIG. 15. Two kite-shaped objects (DKn).

TABLE 3

Comparison of different $n_{L}$ in the tests.

\begin{tabular}{rccrcrccc}
\hline \hline & \multicolumn{9}{c}{ MLSM } & \multicolumn{2}{c}{ LSM } \\
\hline \multicolumn{2}{c}{ Test 1 } & \multicolumn{2}{c}{ Test 2 } & \multicolumn{2}{c}{ Test 3 } & \multicolumn{2}{c}{ Test 2 } \\
$n_{L}$ & Pts. & Time (sec.) & Pts. & Time (sec.) & Pts. & Time (sec.) & Pts. & Time (sec.) \\
\hline 33 & 114 & 0.61 & 128 & 0.67 & 138 & 0.72 & 1089 & 5.98 \\
65 & 235 & 1.29 & 257 & 1.40 & 266 & 1.43 & 4225 & 24.07 \\
129 & 483 & 2.77 & 517 & 2.96 & 516 & 2.95 & 16641 & 99.16 \\
257 & 989 & 5.60 & 1037 & 5.81 & 1016 & 5.75 & 66049 & 396.90 \\
\hline
\end{tabular}

equations that have been solved in each test is around $\zeta_{0} n_{L}$ with $\zeta_{0} \approx 4$, and this further verifies our results in section 2 .

4. Concluding remarks. A novel multilevel linear sampling method (MLSM) is investigated in detail for reconstructing scatterers from far-field measurements. Both theoretical analysis and numerical experiments demonstrate the asymptotically optimal computational complexity of the MLSM. The new method is mainly a new implementation of the linear sampling method (LSM) for inverse scattering problems. It can significantly reduce the computational cost of LSM without any deterioration in quality of the reconstructed scatterers. Exactly as for LSM, MLSM can be applied equally to sound-soft obstacle scattering as well as inverse acoustic sound-hard obstacle scattering, inverse electromagnetic obstacle scattering, and the factorization method in inverse scattering problems (see [11]).

Acknowledgment. The authors would like to thank the two anonymous referees for their constructive and thoughtful comments which have led to a significant improvement of the results and presentation of the current work. 


\section{REFERENCES}

[1] T. Arens, Why linear sampling works, Inverse Problems, 20 (2004), pp. 163-173.

[2] R. Aramini, M. Brignone, and M. Piana, The linear sampling method without sampling, Inverse Problems, 22 (2006), pp. 2237-2254.

[3] F. Cakoni and D. Colton, Qualitative Methods in Inverse Scattering Theory, Springer-Verlag, Berlin, 2006.

[4] D. Colton, H. Haddar, And M. Piana, The linear sampling method in inverse electromagnetic scattering theory, Special section on imaging, Inverse Problems, 19 (2003), S105-CS137.

[5] D. Colton And A. Kirsch, A simple method for solving inverse scattering problems in the resonance region, Inverse Problems, 12 (1996), pp. 383-393.

[6] D. Colton and R. Kress, Integral Equation Method in Scattering Theory, John Wiley and Sons, New York, 1983.

[7] D. Colton And R. Kress, Inverse Acoustic and Electromagnetic Scattering Theory, 2nd ed., Springer-Verlag, Berlin, 1998.

[8] D. Colton And R. KRess, Using fundamental solutions in inverse scattering, Inverse Problems, 22 (2006), pp. R49-R66.

[9] D. Colton, M. Piana, And R. Ротthast, A simple method using Morozov's discrepancy principle for solving inverse scattering problems, Inverse Problems, 13 (1997), pp. 14771493.

[10] A. KIRSCH, An Introduction to the Mathematical Theory of Inverse Problems, Springer-Verlag, New York, 1996.

[11] A. Kirsch and N. Grinberg, The Factorization Method for Inverse Problems, Oxford University Press, Oxford, UK, to appear.

[12] R. Pотthast, A survey on sampling and probe methods for inverse problems, Inverse Problems, 22 (2006), pp. R1-R47.

Copyright (c) by SIAM. Unauthorized reproduction of this article is prohibited. 